\title{
TRAVAUX ORIGINAUXX
}

\section{Possibilité de diagnostic sérologique de la maladie de Newcastle sur le cadavre}

\author{
par J. RAMISSE et E. RAKOTONDRAMARY
}

\begin{abstract}
RÉSUMÉ
Pour diagnostiquer post-mortem la maladie de Newcastle nous nous sommes servis de trois techniques:

L'immunodiffusion et l'hémagglutination n'ont pas donné de résultats positifs et spécıfiques.

L'inhibition de l'hémagglutination nous a fourni des résultats intéressants. Les titres IHA deviennent positifs à partir du 5-6e jour après l'infection. La réaction peut-être pratiquée avec le caillot sanguin hémolysé.
\end{abstract}

Le diagnostic nécropsique de la maladie de Newcastle est parfois délicat. Les lésions hémorragiques du ventricule succenturié, de l'intestin, ou des coecums peuvent être inconstantes, très discrètes, ou confondues avec une irritation parasitaire. L'examen histopathologique du cerveau montre assez souvent une hyperémie congestive, mais ceci ne permet pas de conclure catégoriquement.

C'est pourquoi il faut faire appel, pour compléter les observations d'autopsie, aux techniques virologiques ou sérologiques. Le choix de la technique ou des modalités pratiques peut varier selon qu'il s'agit de sujets vivants (malades et porteurs), ou morts.

En vue de préciser le diagnostic post-mortem, nous avons expérimenté simultanément trols tests: I'hémagglutination (HA), l'ınhibition de l'hémagglutınatıon (IHA), et l'immunodıffusion.

Pour I'IHA nous avons apporté une modification : étant donné l'impossıbılıté de recueillir du sérum sur le cadavre, nous avons pensé à utiliser le caillot sanguin après hémolyse en eau distillée. Des vérifications expérimentales nous ont montré que cette technıque était valable. Nous avons pour cela examiné des caillots prélevés sur des poulets morts d'infection naturelle, mais surtout provenant de poulets inoculés expérimentalement et sur lesquels nous avons fait plusieurs fois des prises de sang.

Ces tests échelonnés pendant l'évolution de la maladie nous ant permis de voir les limites dé leur application, ainsi que la date d'apparition des résultats positifs. Nous avons pu en déduire des conclusions pour le diagnostic post-mortem de la maladie.

Les prélèvements recueillis ont été utilisés de la manière suivante :

- Pour la réaction d'hémagglutination directe, nous nous sommes servis du caillot sanguin hémolysé, du contenu de l'œil, d'extrait tissularre de rate, de rein, de cerveau, de poumon. Le caillot sanguin récolté sur le cadavre ou provenant de prises de sang sur des malades, est trituré au mortier en présence d'eau distıllée ( 1 volume de sang, 3 ou 4 volumes d'eau). La suspension est ensuite centrifugée à faible vitesse pour élıminer les débris globulaires. Le surnageant équivaut approximativement au sang dilué au $1 / 4$ ou au $1 / 5^{\mathrm{e}}$. Les extraits tissulaires sont préparés en broyant les organes au mortier en présence d'eau physıologique ou d'eau distillée. La suspension est centrifugée. On garde le surnageant qui est à la dilution $1 / 5^{\mathrm{e}}$ au $1 / 10^{\mathrm{e}}$. 
- Pour l'inhibition de l'hémagglutination nous n'avons employé que le caillot hémolysé.

- Pour l'immunodiffusion, nous avons choisi les broyats ou suspensions de rate, rein, cerveau ef poumon. Les broyats s'obtiennent en hachant finement les viscères à lạ lame de rasoir, puis en les triturant si nécessaıre au mortier. Les suspensions correspondent aux broyats dilués en tampon physiologique.

Nou' ávóns opéré avec un virus moyennement virulent, et faiblement hémagglutinant, ainsi. qu'avec un virus très hémagglutinant pour I'IHA.

\section{Résultats obtenus avec l'immunodiffusion.}

II existe des anticorps spécifiques précipitants anti-Newcastle dans un certain pourcentage de sérums de poulets infectés ou hyperimmuns (DARBYSHIRE-2, LANCASTER-3). Nous avons recherché l'antigène correspondant dans les broyats tıssulaires de sujets infectés.

1) Méthode : L'antisérum précıpitant provient de poules hyperımmunisées. Le mélange des sérums individuels possède un titre IHA de 1/8192. La diffusion se fait en plaque d'agar à la température du Laboratoire. Les broyats à examiner sont disposés dans les cuvettes périphériques entourant la cuvette centrale contenant l'antısérum. Nous ajoutons un témoin positif : du liquide allantoïdien virulent, et un négatif : du liquide allantordien normal. Nous avons varié les tampons, les $\mathrm{pH}$, la qualité de l'agar.

2) Résultats : Le témoin posttıf donne en 34 jours une ligne nette de précipitation. Le liquide allantaldien normal ne réagit pas. Les broyats ef suspensions d'organes provenant de poulets infectés ne nous ont jamas donné de réaction positive. Pourtant, dans certains cas, les organes ont été prélevés assez précocement après l'infection (4-5 j.). A cette date il n'y avait pas encore d'anticorps inhibiteurs de l'HA dans le sang. A noter que la diffusion des pigments sanguins autour des cuvettes ne facilite pas la lecture.

\section{Résultats obtenus avec l'hémagglutination directe.}

Le pouvoir hémagglutinant spécifique est fonction de la quantité de virus contenu dans les tissus: Selon. LANCASTER (3), les organes les plus riches en virus sont les poumons, la rate, le cerveau. Outre ces organes, nous avons également examiné le caillot hémolysé et le contenu oculaire.

1) Méthode. Les extraits et liquides bıologiques suspects sont dilués"en tampon physiologique, de $1 / 2$ en $1 / 2$, à partir du $1 / 8^{\mathrm{e}}$ ou du $1 / 10^{\mathrm{e}}$. On ajoute un égal volume d'une suspension fraîche d'hématiés de poule à 0,5 p. 100 . La sédimentation se falt en $2-3 \mathrm{~h}$ à $+4{ }^{\circ} \mathrm{C}$ ou à $20^{\circ} \mathrm{C}$.

2) Résultots. Ils sont très peu significatıfs. Avec le sang hémolysé ( 168 examens), quel que soit le stade d'évolution de la maladie, nous n'avons jamais observé d'hémagglutination à partır du $1 / 8$. Avec l'extrait de rate, la réaction est à peu près régulièrement négative, exception faite de quelques échantillons agglutınont jusqu'au $1 / 20^{\circ}$. Etant donné l'ınconstance, d'une réaction positive, nous ne pouvons pas la considérer comme spécifique. Avec les autres extraits organiques, les résultats ne sont pas fidèlement reproductibles. De plus les extraits d'organes normaux non infectés donnent assez souvent des réactions positives (notamment l'extrait de rein, et le contenu oculaire).

\section{Résultats obtenus avec l'inhibition de l'hémag- glutination.}

Nous avons pratiqué cette réaction avec le caillot hémolysé.

1) Méthode ef conditions d'application. Le sang hémolysé est utilisé le jour même, ou conservé en chambre froide. On peut se servir également de sang hémolysé par congélation à $-30^{\circ} \mathrm{C}$. Nous avons remarqué que les hématies sédimentaient mieux dans le sang hémolysé que dans le sérum.

Nous titrons le pouvoir hémagglutınant du virus, et le même jour, nous faisons la réaction d'IHA. Les dilutions sont faites en tampon physiologique de $\mathrm{pH} 7-7,2$.

Le schéma de la réaction est indıqué dans le tableau 1.

Pour que la réaction d'IHA soit correctement réalisée, un certain nombre de conditions sont à respecter : 
TABLEAU $\mathrm{N}^{\circ} \mathrm{I}$

Protocole de l'inhibition de 1 'hémagglutination

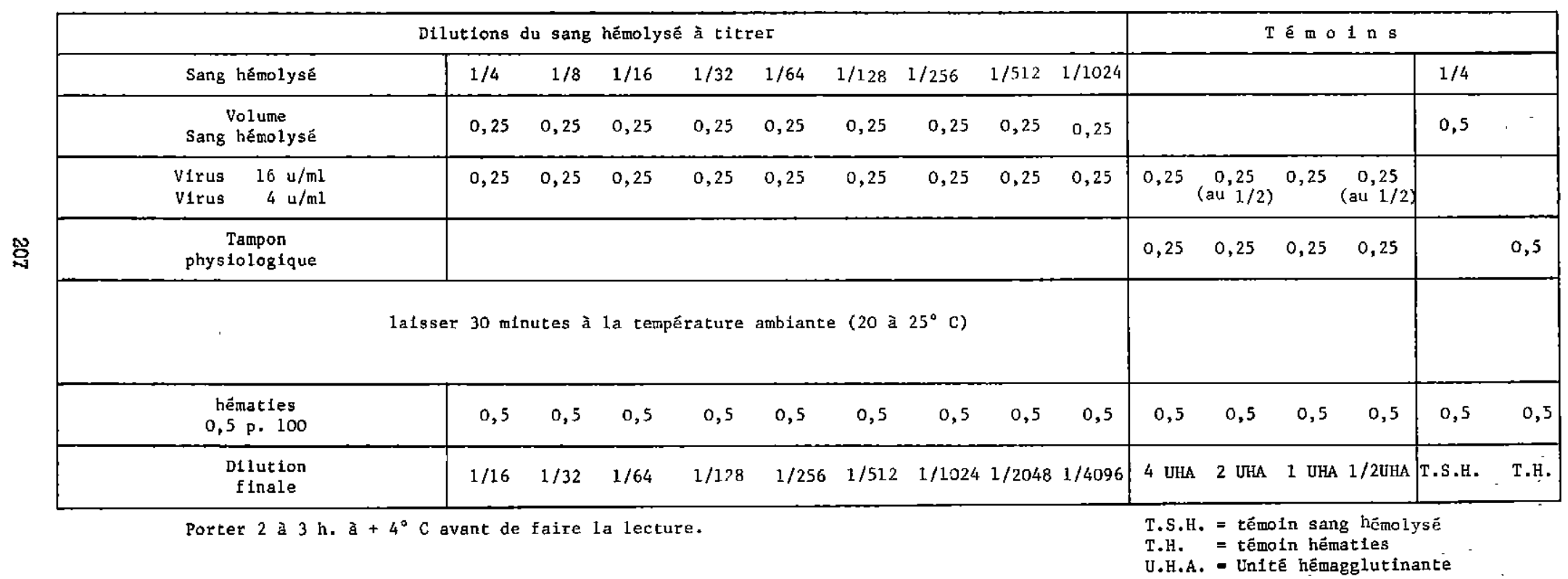


a) Sélection d'hématies agglutinables.

Toutes les hématies de poules ou de poulets ne sont pas également agglutinables par le virus de Newcastle. II existe des différences selon les sujets, et aussi selon l'âge.

- Différences selon les sujets: Dans un groupe de poulets du même âge, les uns ont des hématies très agglutinables, les autres des hématies peu ou pas agglutinables. La proportion est aux environs de 50 p. 100 . Avec un même virus, le titre hémagglutınant a varié de 8 UHA à 4.096 UHA, selon les hématies utilisées, toutes les conditıons étant identrques par ailleurs. Cette constatation impose de bien sélectionner les poulets donneurs avant d'entreprendre les réactions.
- Différences selon l'âge : Les hématies de poulets de plus de 3-4 mois ou de poules adultes sont plus agglutinables que celles de jeunes poulets. La vaccination anti-Newcastle ne semble pas intervenir pour modifier l'agglutinabilité.

Avec un même virus nous avons testé plusieurs lots d'hématies provenant:

- de poulets de 1 mois non vaccinés,

- de poulets de 2 mois vaccinés,

- de poulets de 4 mois vaccinés,

- de poulets de 6 mois hyperimmunisés.

Les résultats sont relatés tableau no $\|$ : Agglutinabilité des hématies.

TABLEAU $N^{\circ} I I$

Agglutinabilitié des hểmaties

\begin{tabular}{|c|c|c|c|c|}
\hline Dilutions virus $\mathrm{Age}$ des poulets & $\begin{array}{c}\text { Poulets d' } 1 \text { mols } \\
\text { non vaccinés }\end{array}$ & $\begin{array}{c}\text { Poulets de } 2 \text { mols } \\
\text { vaccinés }\end{array}$ & $\begin{array}{c}\text { Poulets de } 4 \text { mois } \\
\text { vacclnés }\end{array}$ & $\begin{array}{l}\text { Poulets de } 6 \text { mois } \\
\text { hypérimonunisés }\end{array}$ \\
\hline $1 / 1$ & - & 1 & - & - \\
\hline $1 / 8$ & 4 & 1 & 1 & 1 \\
\hline $1 / 16$ & - & 2 & 1 & - \\
\hline $1 / 32$ & 3 & - & - & - \\
\hline $1 / 64$ & 2 & 1 & - & - \\
\hline $1 / 128$ & 1 & - & 1 & I \\
\hline $1 / 256$ & - & 2 & - & 1 \\
\hline $1 / 512$ & - & - & - & 1. \\
\hline $1 / 1024$ & - & - & 1 & 1 \\
\hline $1 / 2048$ & - & 1 & 3 & - \\
\hline $1 / 4096$ & - & - & 3 & 4 \\
\hline
\end{tabular}

b) Comparaison des hématies fraîches et formolées.

Les hématies fraîches sont conservées sur Alsever, ou en suspension à 0,5 p. 100 en tampon physiologique, à $+4 \mathrm{o}$ C. Les hématies formolées sont préparées selon la technique de CSIZMAS (1).

Nous avons comparé l'agglutinabilité d'hématies fraîches et formolées provenant de la même prise de sang. A piusieurs reprises nous avons constaté que les hématies formolées étaient beaucoup moins agglutınables par le virus de Newcastle que les hématies fraîches : les différences allaient de 64 UHA avec les hématies formolées à 2.048 UHA avec les hématies fraîches. Par la suite nous avons donc travailié avec les hématies fraîches seulement.

c) Choix d'un virus suffisamment hémoggluhnant.

Les hématies sont irrégulièrement agglutinables par un même virus, les variations ne sont pas moins importantes avec diverses souches de virus.

Nous avons comparé plusieurs fois, avec divers lots d'hématies, les titres hémagglutinants des souches virales en notre possession. Nous rapportons les résultats moyens - Tableau III : Titre HA des virus examinés. 


\section{TABLEAU $N^{\circ}$ III}

Titre HA des virus examinés (1ecture après $2 \mathrm{~h}$ à $+4^{\circ} \mathrm{C}$ )

Souche virulente Malgache

Souche Ambatolampy

Souche passée sur perdrix

Souche Maurice

Souche Pakistan

Souche Mukteswar mésogénique

Pour les épreuves d'IHA nous avons utilisé le virus-vaccin mésogénique qui est de loin le plus hémagglutinant.

d) Sédimentotion des hématies dans les sérums ef les sangs hémolysés.

Pour que les réactions d'HA ef IHA aient une signification, il ne faut pas que ces hématies soient spontanément agglutinées dans le sérum ou le sang hémolysé, même aux plus faibles dilutions utilisées.

Or il arrive qu'avec certains sérums, les hématies soient agglutinées, et ceci jusqu'au $1 / 8 \mathrm{e}$ ou $1 / 16 \mathrm{e}$. Les réactions d'HA et IHA risquent d'être perturbées. Ce pouvoir hémagglutinant non spécıfique varie selon les sérums. Il existe aussi bien avec les sérums de poulets neufs qu'avec ceux de poulets vaccinés, ou hyperimmunisés, ou malades. L'inactivation par chauffage $30 \mathrm{mi}-$ nutes à $56^{\circ} \mathrm{C}$ ne modifie pas régulièrement cette propriété.

Par contre dans le sang hémolysé, à la même dilution que le sérum, les hématies ne sont pas spontanément agglutinées. Soit un sérum dilué au $1 / 16^{e}$ qui hémagglutine d'une manière non spécifique, le sang représentant approximativement le double de volume du sérum, on peut considérer qu'au sérum dilué au $1 / 16^{e}$ correspond le sang hémolysé dilué au $1 / 8 \mathrm{e}$. Nous avons constaté que le sang hémolysé au $1 / 8 \mathrm{e}$ n'hémagglutıne pas, alors que le sérum au $1 / 16^{\mathrm{e}}$ peut-parfois agglutiner. La sédimentation paraît donc meilleure dans le sang hémolysé que dans le sérum.

D'autre part nous n'avons pas constaté d'inhibiteurs non spécifiques de l'hémaggiutination dans les sérums ef sangs hémolysés normaux. Ces réactifs, qu'ils soient traités ou non au périodate de poiasse n'inhibent pas l'hémagglutination, tout au moins aux dilutions utilisées.

2) Résultats de l'IHA avec le sang hémolysé.

Les tests expérimentaux ont été réalısés sur des poulets neufs inoculés avec des doses variables de virus Newcastle virulent. Nous avons introduit le virus par la voie sous cutanée, ou par la voie nasale. Nous pensions que l'infection par voie nasale à faible dose se rapprochait davantage du mode d'infection naturelle. La DLM du virus est environ $10^{-6}$ pour des poulets de 2-3 mois. La durée d'évolution de la maladie est en moyenne de 5 à 7 jours.

Pour comparaison nous avons également recherché le titre $\mathrm{HHA}$ de sangs hémolysés provenant de poulets vaccinés.

Les poulets infectés expérimentalement ont été divisés en quatre groupes :

- deux groupes inoculés par voie sous cutanée :

L'un avec une dose massive de virus $(1 \mathrm{ml}$ de virus pur).

L'autre avec une dose faible de virus $(1 \mathrm{ml}$ de virus d̀ $10^{-6}$ ).

- deux groupes inoculés par voie nasale:

L'un avec une dose élevée de virus (1 goutte de virus pur).

L'autre avec une dose plus fable (10 vaporisations de virus à $10^{-2}$ ).

Les titres inhıbiteurs de l'HA ont été évalués sur tous les poulets avant l'inoculation, et sur un certain pourcentage au fur et à mesure de l'évolution de l'infection. Quelques poulets ont succombé à la ponction cardiaque en cours de maladie.

Les résultats des titrages sont classés dans le tableau IV. Pour chaque groupe de poulets, nous indiquons en fonction du temps :

- Le nombre de morts ( $P$. C. = mort par ponction cardiaque) ;

- Le nombre de sangs hémolysés testés ;

- le nombre de positifs à l'IHA ;

- Les titres IHA minimum et maximum ;

- Le nombre de poulets résistants.

D'après ces résultats, Il ressort que :

- Un certain pourcentage de poulets résistent à l'infection expérimentale, même iorsque la 


\begin{tabular}{|c|c|c|c|c|c|c|c|c|c|c|}
\hline \multirow{4}{*}{ sวนeาstsagx } & $\begin{array}{l}Z 6 \mathrm{~T} 8 / \mathrm{I} \\
7 \mathrm{Z0 \tau} / \mathrm{L}\end{array}$ & $\begin{array}{l}\tau I S / \tau \\
8 Z \tau / \tau\end{array}$ & $\begin{array}{l}8 Z \mathrm{~T} / \mathrm{T} \\
9 \mathrm{~T} / \mathrm{T}\end{array}$ & $Z \varepsilon / I-9 \tau / \tau$ & & . & & 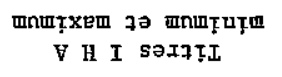 & \multirow{4}{*}{ oz } & \multirow{4}{*}{$\begin{array}{c}\text { suogizesțioden } \\
\text { OI } \\
\text { z-0I } \\
\text { snIts } \\
\text { ageseu }\end{array}$} \\
\hline & $L$ & $\varepsilon$ & 8 & $\varepsilon$ & 0 & 0 & 0 & sfțțsod & & \\
\hline & $L$ & $\varepsilon$ & 8 & 5 & 5 & $\varsigma$ & snot & sפ7827 & & \\
\hline & 0 & $\left(\cdot \nu^{\prime} \mathrm{d}\right) \mathrm{I}$ & $\varepsilon$ & $(\cdot 5 \cdot d){ }^{T}$ & 0 & 0 & & s7jour & & \\
\hline \multirow[t]{4}{*}{ słuezsṭsəِx } & $\begin{array}{l}26 \mathrm{LI} / \mathrm{I} \\
8 \mp 0 Z / \mathrm{I}\end{array}$ & $\begin{array}{l}7 Z 0 L / \tau \\
9 \subseteq Z / \tau\end{array}$ & $Z \mathrm{I} \varsigma / \mathrm{I}-Z E / \mathrm{L}$ & $9 \zeta Z / I-9 I / I$ & $9 \tau / \tau$ & $9 I / I$ & & 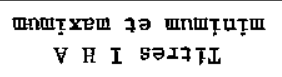 & \multirow{4}{*}{$\varepsilon I$} & \multirow{4}{*}{$\begin{array}{l}\text { ażno8 I } \\
\text { xnd snxth } \\
\text { •ə[egeu }\end{array}$} \\
\hline & 9 & 9 & $L$ & $L$ & $\tau$ & $\mathbf{I}$ & 0 & sftutsod & & \\
\hline & 9 & 9 & $L$ & $L$ & 9 & $L$ & snoz & 8297827 & & \\
\hline & 0 & 0 & I & 0 & $\rightarrow$ & $(\cdot 0 \cdot d) z$ & & s7.10س & & \\
\hline \multirow{4}{*}{$\begin{array}{c}\text { s7ueqsțsag } \\
0 Z / 6\end{array}$} & $\begin{array}{r}\eta Z 0 \tau / I \\
Z \tau S / I\end{array}$ & & $\begin{array}{l}Z \mathrm{IIS} / \mathrm{L} \\
9 S Z / \mathrm{I}\end{array}$ & $\tau \varepsilon / \tau-9 \tau / \tau^{\prime}$ & $9 \tau / \tau$ & & & 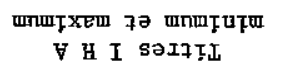 & \multirow{4}{*}{$0 z$} & \multirow{4}{*}{ 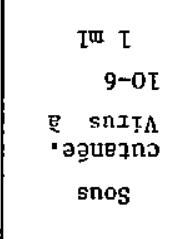 } \\
\hline & $\varepsilon$ & & $\varepsilon$ & II & 9 & 0 & 0 & sэTftsod & & \\
\hline & $\varepsilon$ & & $\varepsilon$ & II & $\eta[$ & 6 & sno7 & sazsaz & & \\
\hline & 0 & & 0 & 8 & $\varepsilon$ & 0 & & $87 x 01$ & & \\
\hline \multirow{4}{*}{$\begin{array}{c}\text { 8جuefsts } \\
0 z / L\end{array}$} & $z \mathrm{~L} / \mathrm{L}-+79 / \mathrm{L}$ & $8 Z \tau /$ I-†9/ & $\tau \tau \varsigma / \mathrm{I}-\tau \varepsilon / \tau$ & $9 \varsigma Z / \tau-\not 9 / \tau$ & $79 / \tau-9 I / \tau$ & & & 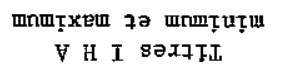 & \multirow{4}{*}{$0 z$} & \multirow{4}{*}{ 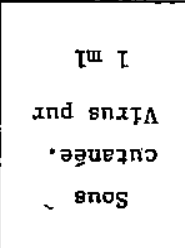 } \\
\hline & 9 & $\varepsilon$ & or & $L$ & 8 & 0 & 0 & SÆItTSOd & & \\
\hline & 9 & $E$ & OI & $L$ & II & 5 & sno7 & 897527 & & \\
\hline & $\varepsilon$ & 0 & $z$ & $\varepsilon$ & $\varsigma$ & 0 & & s7xow & & \\
\hline $\begin{array}{l}\text { s7uențas } \\
\text { sina! }\end{array}$ & 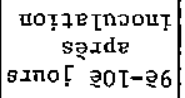 & $\begin{array}{c}\text { uoțegnวout } \\
\text { səxde } \\
\text { mo! } 98\end{array}$ & 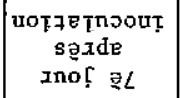 & $\begin{array}{c}\text { uoțfe [noout } \\
\text { səIde } \\
\text { xno! } 29\end{array}$ & 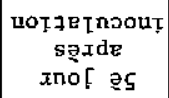 & 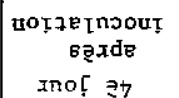 & $\begin{array}{c}\text { UOIZE LnכOUT } \\
\text { ZUEAV }\end{array}$ & \multirow[t]{2}{*}{ słe] [ns } & \multirow{2}{*}{$\begin{array}{c}\text { spajnod } \\
\text { ap } \\
\text { aIquoN }\end{array}$} & \multirow{2}{*}{ 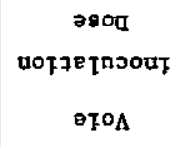 } \\
\hline \multicolumn{7}{|c|}{ әтре โеш } & & & & \\
\hline
\end{tabular}

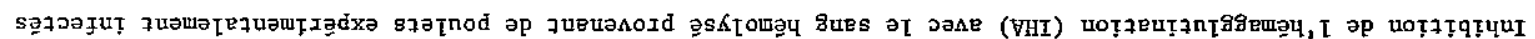
$\Lambda I_{0} N$ กVमr ItgL 
dose inoculée est forte. Pourtant ces poulets n'avaient pas d'anticorps IHA avant I'inoculation. La souche de virus ne paraît pas extrêmement virulente.

- Les poulets résistants ont un titre en anticorps IHA très élevé. On peùt remarquer d'ailleurs que l'inoculation par voie nasale permet un développement nettement plus marqué des anticorps IHA.

- La mortalité maxıma se situe vers le 56e jour après l'ınoculation. La durée d'évolution de la maladie varie peu quelles que soient la voie d'inoculation, ou la dose inoculée.

- Les anticorps IHA n'apparaissent qu'à partir du 5 e jour (inoculation sous cutanée), ou fe jour (inoculation voie nasale). C'est-à-dire au moment de la mortalité maxima. Ceci donne à penser que lors d'évolution suraigué (souche très virulente), il n'y a pas d'anticorps décelables, et que par conséquent le dıagnostic ne peut dans ce cas être établi par IHA. Le diagnostic parl HA post-mortem ne pourrait être fait, selon notre expérimentation, que si l'évolution de l'infection dure au moins 5 jours.

D'autre part pour appliquer valablement cette méthode, il faut savoir si les sujets ont été vaccinés et à quelle date. La vaccination fait apparaître des anticorps IHA. Nous avons évalué ces anticorps sur un lof de poulets vaccinés depuis 15 jours avec un virus vivant mésogénique inoculé en sous-cutanée. Les titres IHA allaient de $1 / 128$ è $1 / 1.024$ e. Si l'on examine des poulets suspects mais ayant été vaccinés auparavant, le titre en anticorps IHA ne peut guère avoir de signification diagnostique.

\section{CONCLUSIONS}

Nous avons applıqué l'hémagglutination, l'immunodıffusion, et l'inhibition de l'hémagglutınation au diagnostic de la maladie de Newcastle.

Les poulets soumis à l'examen ont été infectés expérımentalement. Le virus était moyennement virulent.

L'immunodiffusion avec des broyats tissularres ne nous a donné que des résultats négatifs.

L'hémagglutination avec des extraits d'organes et de sang hémolysé n'a fourni aucun renseignement utile, car elle est demeurée négative ou non spécifique.

Par contre, avec l'inhibition de l'hémagglutınation pratiquée sur les sangs hémolysés, nous avons obtenu des réponses significatives et constantes. Avant l'inoculation, I'lHA est habituellement négative. Les anticorps spécifiques apparaissent vers le 5-6e jours après l'infection. Ills sont décelables au moment de la mortalité maxima et peuvent servir de ce fait au diagnostic post-mortem sous réserve que les poulets n'aient pas été vaccinés.

Les limites d'application de l'IHA découlent des faits suivants :

- Si la mortalité est précoce (souches très virulentes) il n'y a pas d'anticorps. Peut-être dans ce cas est-il possıble de faire appel àl hémaggiutination?

- Les sujets vaccinés élaborent des anticorps IHA qui ne peuvent être différenciés des antıcorps induits par l'ınfection.

\section{Institut d'Elevage ef de Médecine vétérinaire des Pays tropicaux. Laboratoire central de l'Elevage de Tonanarive.}

\section{SUMMARY}

\section{Post-mortem serological diagnosis of Newcastle Disease}

Three methods have been used for the diagnosis of Newcastle Disease :

- Immunodiffusion and hemagglutination did not show positive and specific results.

- Hemagglutination inhibition test gave some interesting results. HIT became positive from 5 or 6 th day after infection. The reaction can be carried out with the hemolysed blood clot. 


\section{RESUMEN}

\section{Posibilidad de diagnóstlco serologitco post-mortem} de la enfermedad de Newcastle

Se utilizaron tres técnicas para el diagnóstico de la enfermedad de Newcastle :

La immunodifusión y la hemaglutinación no daron resultados positivos y especificos.

La inhibición de la hemaglutinación es interesante en cuanto a sus resultados. Los títulos de la inhibición de la hemaglutinación se hacen positivos a partir del $5^{\circ} \circ$ del $6^{\circ}$ día despuès de la infección. Se puede efectuar la reacción con el coágulo hemolizado.

\section{BIBLIOGRAPHIE}

1. CSIZMAS (L.). - Préparation d'hématies formolées. Proc. Soc. Exp. Biol. and Med., 1960, 103, 157.

2. DARBYSHIRE. - La technique de diffusion en gel dans l'étude des virus importants en Médecine Vétérinaire. Vét. Bull, 1964, 34, 699.

3. LANCASTER, — Diagnostic de la maladie de Newcastle. Vét. Bull., 1964, 33, 347. 